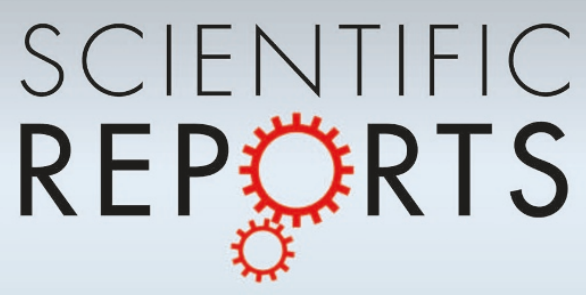

open One-Pot Synthesis of

SUBJECT AREAS:

ELECTROCATALYSIS

SYNTHESIS OF GRAPHENE

Received

10 January 2014

Accepted

3 March 2014

Published

28 March 2014

Correspondence and requests for materials should be addressed to

Z.H.Y. (yaozh@ tsinghua.edu.cn) or X.G.Z. lazhangxg@

163.com)

* These authors contributed equally to this work.

\section{Graphene-Supported Monodisperse Pd Nanoparticles as Catalyst for Formic Acid Electro-oxidation}

\author{
Sudong Yang 1,3,4*, Jing Dong ${ }^{2 *}$, Zhaohui Yao², Chengmin Shen', Xuezhao Shi', Yuan Tian', \\ Shaoxiong Lin' \& Xiaogang Zhang ${ }^{3}$
}

\begin{abstract}
'Beijing National Laboratory for Condensed Matter Physics, Institute of Physics, Chinese Academy of Sciences, Beijing 100190, P. R. China, ${ }^{2}$ School of Aerospace, Tsinghua University, Beijing 100084, P. R. China, ${ }^{3}$ College of Material Science and Engineering, Nanjing University of Aeronautics and Astronautics, Nanjing 210016 , P. R. China, ${ }^{4}$ Laboratory of Eco-Materials and Sustainable Technology (LEMST), Xinjiang Technical Institute of Physics \& Chemistry, Chinese Academy of Sciences, Urumqi, 830011 , P. R. China.
\end{abstract}

To synthesize monodisperse palladium nanoparticles dispersed on reduced graphene oxide (RGO) sheets, we have developed an easy and scalable solvothermal reduction method from an organic solution system. The RGO-supported palladium nanoparticles with a diameter of $3.8 \mathrm{~nm}$ are synthesized in $\mathrm{N}$-methyl-2-pyrrolidone (NMP) and in the presence of oleylamine and trioctylphosphine, which facilitates simultaneous reduction of graphene oxide and formation of Pd nanocrystals. So-produced Pd/RGO was tested for potential use as electrocatalyst for the electro-oxidation of formic acid. Pd/RGO catalyzes formic acid oxidation very well compared to Pd/Vulcan XC-72 catalyst. This synthesis method is a new way to prepare excellent electrocatalysts, which is of great significance in energy-related catalysis.

(1) alladium (Pd) nanoparticles play an important role in the hydrogenation of organic compounds ${ }^{1}$. They also serve as the primary catalyst for the reduction of pollutants emitted from automobiles ${ }^{2}$ and facilitate a range of coupling reactions $s^{3-5}$. Owing to Pd's higher abundance and proven electro catalytic effect on small organic molecules, it has the potential to emerge as a substitute for Pt especially in the development of direct formic acid fuel cells (DFAFCs) ${ }^{6-9}$. It is, however, essential to further maximize the activity of Pd and minimize the use of Pd. There is a most urgent demand for developing methods to load high-activity Pd nanostructures on the surface of supporting nanomaterials at a low cost, with a large surface area and good electrical conductivity. This will not only to maximize the availability of electrocatalyst surface area for electron transfer but also to provide better mass transport of reactants to the electrocatalyst ${ }^{10}$.

Graphene nanosheets have opened a new avenue for utilizing 2D carbon materials as catalyst support due to the high conductivity, huge specific surface area, and low fabrication cost $\mathrm{t}^{11-13}$. One of popular methods for synthesizing graphene supported metal NPs catalysts is the chemical reduction of metal salts in the presence of graphene oxide (GO) in aqueous solution ${ }^{14-17}$. Using this method, metal NPs usually have broader distributions in size and shape on the graphene surface. To overcome this problem, the synthetic approach in organic phase is greatly potential. It offers good controls on dispersity, crystallization, size, and shape for metal NPs on the graphene sheets.

Previous research reported that GO can form dispersions with long-term stability in $N$-methyl-2-pyrrolidone $(\mathrm{NMP})^{18}$. The high boiling point $\left(202^{\circ} \mathrm{C}\right)$ of NMP facilitates its use as a solvent to reduce a graphene oxide suspension by solvothermal reduction ${ }^{19-20}$. Herein, we report a new solvothermal reduction method. In this onepot synthesis method, NMP serves as both dispersing and reducing agent for both the metal precursor and GO, facilitating the formation of monodisperse Pd nanoparticles on RGO surface. To the best of our knowledge, such excellent reduction performance of NMP has rarely been observed on graphene-based supports. As-produced Pd/ RGO was tested for its potential use as electrocatalyst for the electro-oxidation of formic acid. Pd/RGO catalyzes formic acid oxidation very well compared to Pd/Vulcan XC-72 catalyst. A schematic sketch of the growth process of $\mathrm{Pd} / \mathrm{RGO}$ composites is shown in Figure 1. 


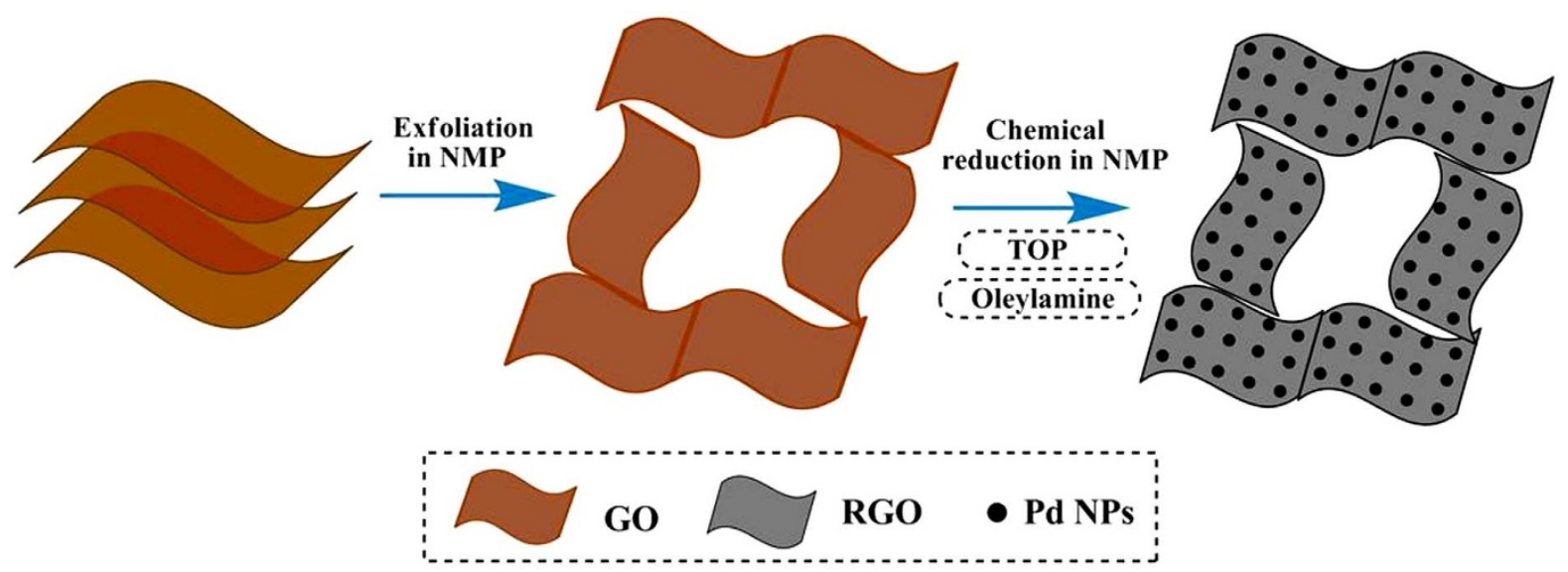

Figure 1 Illustration of the synthesis procedure of the Pd/RGO catalysts. GO is dissolved in NMP and formed uniformly solution; Then Pd(acac) ${ }_{2}$, $\mathrm{OAm}$ and TOP are added into mixed solution and heated to $200^{\circ} \mathrm{C}$ for 2 hours; Finally, monodisperse Pd NPs are formed on the surface of RGO.

\section{Results}

The Pd/RGO composites were prepared by simultaneous reduction of $\mathrm{GO}$ and $\mathrm{Pd}(\mathrm{acac})_{2}$ using NMP as solvent and reducing agent in the presence of TOP and OAm. The morphologies of Pd / RGO were characterized by transmission electron microscopy (TEM). From the low-resolution TEM images of the Pd/RGO (Figure $2 \mathrm{a}, \mathrm{b}$ ), we found that the Pd NPs were uniformly dispersed on the surface of RGO and had a mean diameter of $3.8 \mathrm{~nm}$. Due to the narrow size distribution of our Pd NPs, a self-assembled structure of NPs formed on the surface of the RGO films. High-resolution TEM (HRTEM) analysis (Figure 2c) revealed that the Pd NPs had many grain boundaries and were polycrystalline in structure. The crystal domains within Pd NPs have an interfringe distance of $0.23 \mathrm{~nm}$, which is close to the lattice spacing of the (111) plane in face-centered cubic (fcc) Pd crystal
$(0.223 \mathrm{~nm})$. The structures of GO and Pd NPs loaded on the GO films were analyzed by XRD technology. The X-ray diffraction (XRD) pattern in Figure 2d shows that the typical diffraction peak (002) of GO shifts to a higher angle $\left(2 \theta \approx 25^{\circ}\right)$ after reduction (S1), indicating that the GO reduces partially to graphene and restacks into an ordered crystalline structure. The reflection peaks located at $39.9^{\circ}, 46.3^{\circ}, 67.8^{\circ}$ and $81.6^{\circ}$, correspond to the (111), (200), (220), and (311) planes of an $f c c$ lattice, respectively. This result confirms that an $f c c$ crystalline structure was formed. The diffraction peak (111) at around $39.88^{\circ}$ in Figure $2 \mathrm{~d}$ was found to shift slightly toward lower angles as compared with bulk $\operatorname{Pd}\left(2 \theta=40.12^{\circ}\right.$, JCPDS 00-0050681), indicating that the Pd-Pd interatomic distance increases after formation of the Pd NPs, which is consistent with a previous report $^{21-22}$. The mean crystalline size was estimated to be $\sim 2.1 \mathrm{~nm}$

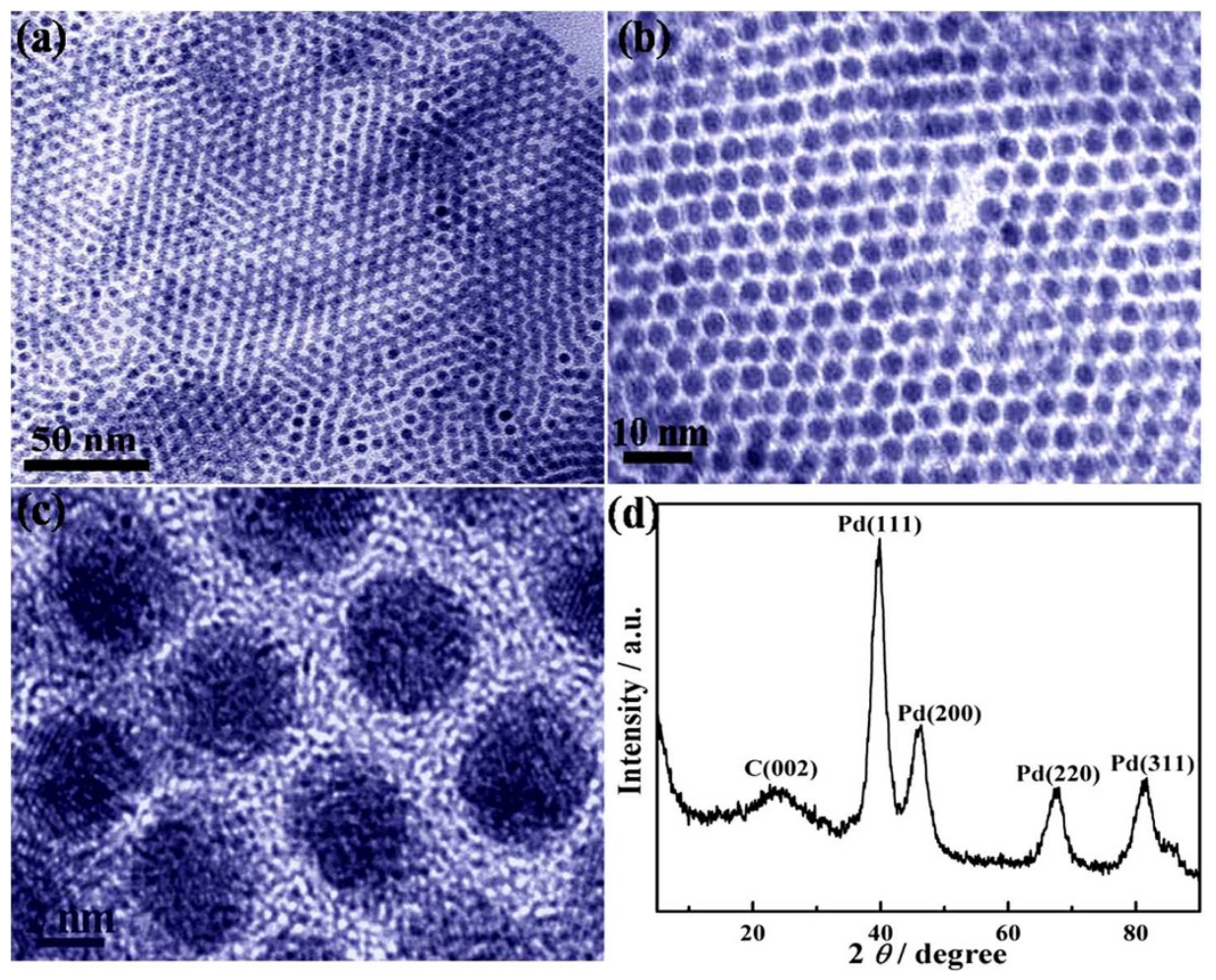

Figure $2 \mid$ TEM and HRTEM images of Pd/RGO composites with $0.4 \mathrm{~mL}$ TOP and $0.5 \mathrm{~mL} \mathrm{OAm}$; (a) and (b) TEM images, (c) HRTEM image; (d) XRD pattern of the Pd/RGO composites with $0.4 \mathrm{~mL}$ TOP and $0.5 \mathrm{~mL} \mathrm{OAm}$. The results indicated that the Pd NPs with a mean diameter of $3.8 \mathrm{~nm}$ were uniformly dispersed on the surface of RGO. Pd NPs formed a self-assembled structure on the surface of the RGO films due to the narrow size distribution. 

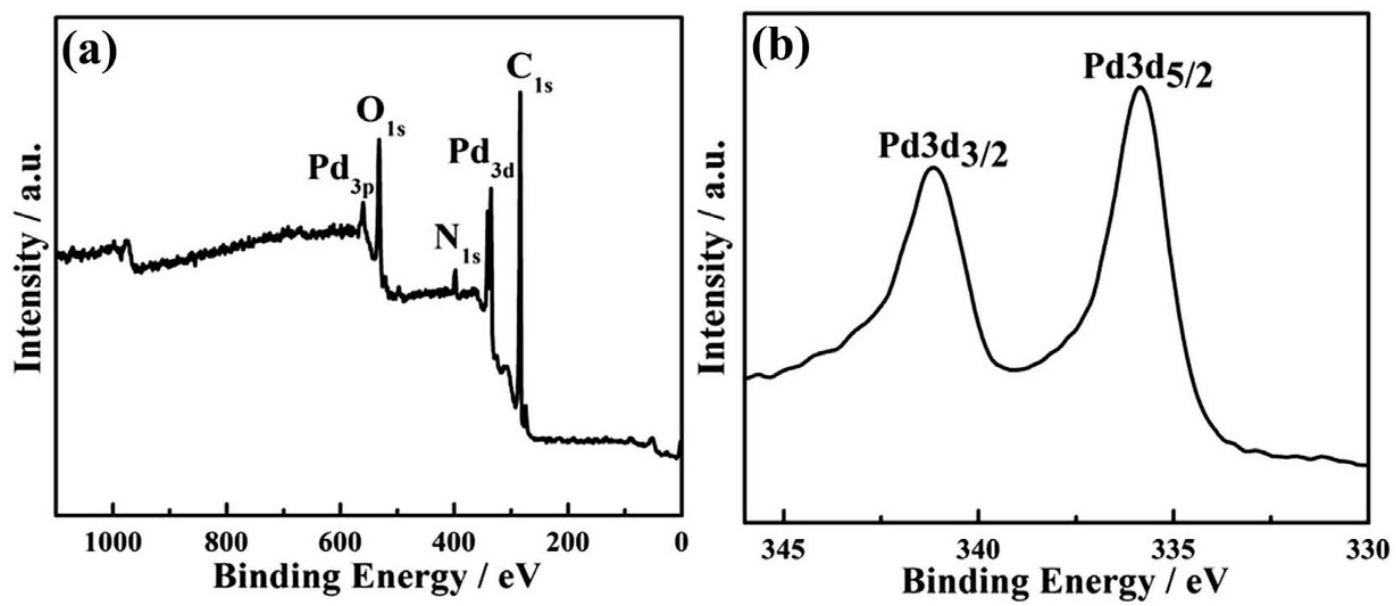

Figure 3 XPS spectra of Pd/RGO composite: (a) XPS full spectrum of Pd/RGO and (b) Pd spectrum. XPS full spectrum analysis indicated only palladium, oxygen, nitrogen and carbon, the absence of phosphorus indicating that no TOP molecules are on the surface of the Pd NPs and RGO. The XPS spectrum of the $\mathrm{Pd}$ spectrum showed double peaks with binding energies at $335.1 \mathrm{eV}$ and $340.4 \mathrm{eV}$, corresponding to $\mathrm{Pd}_{3 \mathrm{~d} 5 / 2}$ and $\mathrm{Pd}_{3 \mathrm{~d} 3 / 2}, \mathrm{respectively}$.

using the Scherrer equation through line broadening of the $\mathrm{Pd}(111)$ peak. This is smaller than the mean particle size of $3.8 \mathrm{~nm}$, measured from TEM, which indicates that the Pd NPs are polycrystalline, in accordance with the HRTEM observation. Obviously, the XRD patterns of the composites prove that NMP, as reducing reagent, can simultaneously reduce GO to RGO and Pd(acac $)_{2}$ to Pd NPs.

To study the reaction parameters that dominate the Pd growth in the current synthesis, we performed a series of control experiments by varying the reactants added in the reaction mixture, and analyzed the Pd NP morphology at various stages of synthesis. Without OAm and TOP in the solution, monodisperse Pd NPs could not be formed on the surface of RGO. Instead, the synthesis yielded random NPs with diameters in the range of about 10-20 nm (S2). It is difficult to control the size and shape of Pd NPs when only OAm or only TOP is present in the reaction solution, indicating that the combination of both OAm and TOP is necessary to form monodisperse Pd NPs.

For all the reaction parameters, using the optimal amount of TOP appears to be the key to successful preparation of monodisperse NPs. TOP can be capped on the surface of metals particles as a surfactant, but it does not stop a particle's growth ${ }^{23}$. As a result, larger and aggregated particles are formed when TOP is used alone. When other experimental conditions were fixed, different amounts of TOP were added before heating. In the absence of TOP, only nanopod-like aggregates with a broad size distribution are produced. Addition of TOP $(0.4 \mathrm{~mL})$ into the typical system results in Pd NPs with good size distribution. If the amount of TOP is increased to $0.6 \mathrm{~mL}$, the final product is composed of aggregated NPs on the RGO layer (S3). Further increasing the molar ratio of TOP/Pd(II) results in random size distribution of Pd NPs due to large steric hindrance of TOP. Therefore, TOP plays the most important role in controlling the size of the Pd NPs.

It became clear that OAm plays significant but different role in preparing monodisperse $\mathrm{NPs}^{24}$. The OAm not only acts as surfactant to stabilize Pd NPs, but also can serve as a middle reducing reagent for making $\mathrm{Pd} \mathrm{NPs}^{25}$. We infer that an appropriate amount of OAm is essential to tentatively stabilize the clusters of Pd and the growth of Pd NPs is synergistically controlled by the TOP and OAm together. Without OAm, random Pd NPs with a high degree of aggregation were formed (Figure S2c), which indicates that OAm is indispensable for stabilizing the colloidal NPs. If too much OAm (e.g., $7 \mathrm{~mL}$ ) was used, however, polydisperse and aggregated NPs were produced (Figure S2d), which suggests that less Pd is released for the nucleation because of the stronger binding of $\mathrm{OAm}^{26-27}$. In order to get highquality Pd NPs, the volume ratio of TOP/OAm was optimized in the range from 0.04 to 0.12 .
In order to study the factors of precursors that dominate the $\mathrm{Pd}$ growth in the typical synthesis, we performed the control experiments by using the different precursors, and analyzed the Pd NP morphology on the RGO surface. Of the three $\mathrm{Pd}$ precursors $\left(\mathrm{Pd}(\mathrm{acac})_{2}, \mathrm{Pd}\left(\mathrm{C}_{2} \mathrm{H}_{3} \mathrm{O}_{2}\right)_{2}\right.$, and $\left.\mathrm{Na}_{2} \mathrm{PdCl}_{4}\right)$ screened under the described synthesis conditions, $\mathrm{Pd}(\mathrm{acac})_{2}$ empirically gave the best-quality Pd NPs. All the large NPs and nanopod-like Pd clusters were obtained when palladium acetate and sodium tetrachloropalladate instead of palladium acetylacetonate were used as the metal precursors (Figure $\mathrm{S} 4 \mathrm{a}, \mathrm{b}$ ). The results from our system suggest that the formation of different shaped metal nanoparticles could be explained by considering the different reaction precursors. Control experiments on the formation of Pd NPs on XC-72 (Figure S5) show a highly non-uniform distribution of larger Pd particles that aggregate significantly in many regions, further confirming that the homogeneous nucleation environment does play a significant role in the case of GO.

To intuitively evaluate the reduction level and determine the composition of the as-prepared $\mathrm{Pd} / \mathrm{RGO}$ composite, X-ray photoelectron spectroscopy (XPS) was utilized. As shown in Figure 3a, The XPS full-spectrum indicates only palladium, oxygen, nitrogen and carbon, the absence of phosphorus indicating that no TOP molecules are on the surface of the Pd NPs and RGO. In Figure 3b, the XPS spectrum of the Pd shows double peaks with binding energies at $335.1 \mathrm{eV}$ and $340.4 \mathrm{eV}$, corresponding to $\mathrm{Pd}_{3 \mathrm{~d} 5 / 2}$ and $\mathrm{Pd}_{3 \mathrm{~d} 3 / 2}$, respectively. Compared to the standard spectra of metal $\mathrm{Pd}^{\circ}$, the binding energies of $\mathrm{Pd}$ increase slightly (from $335.1 \mathrm{eV}$ to $335.9 \mathrm{eV}$ and from $340.4 \mathrm{eV}$ to $341.2 \mathrm{eV}$ ). These results combined with FT-IR spectra confirm that the OAm can be adsorbed on the surface of Pd particles. The composition of the Pd/RGO was prepared only in the NMP solution and analyzed by XPS. The highresolution XPS spectrum of the Pd $3 \mathrm{~d}$ region (Figure S6a) reveals the existence of Pd with binding energies $3 \mathrm{~d}_{5 / 2}=335.1 \mathrm{eV}$ and $3 \mathrm{~d}_{3 / 2}=$ $340.4 \mathrm{eV}$, which are in good agreement with the expected values for $\mathrm{Pd}^{\circ}$. These results confirm that the NMP cannot be adsorbed on the surface of Pd particles. This explanation is consistent with the results of FT-IR spectra data. Although NMP has been applied successfully as the reducing reagent for the synthesis of $\mathrm{Pd}$ nanoparticles, our results indicate it may not end up as a capping ligand for the $\mathrm{Pd}$ nanoparticles. To further confirm that, another experiment was carried out under the same conditions, except that the OAm and TOP were absent. The obtained particles were still not stable in the solution and not ready to settle down as black precipitates. Although the RGO used for XPS analysis had been washed extensively using ethanol and acetone, some residual NMP could be present at the sheet 

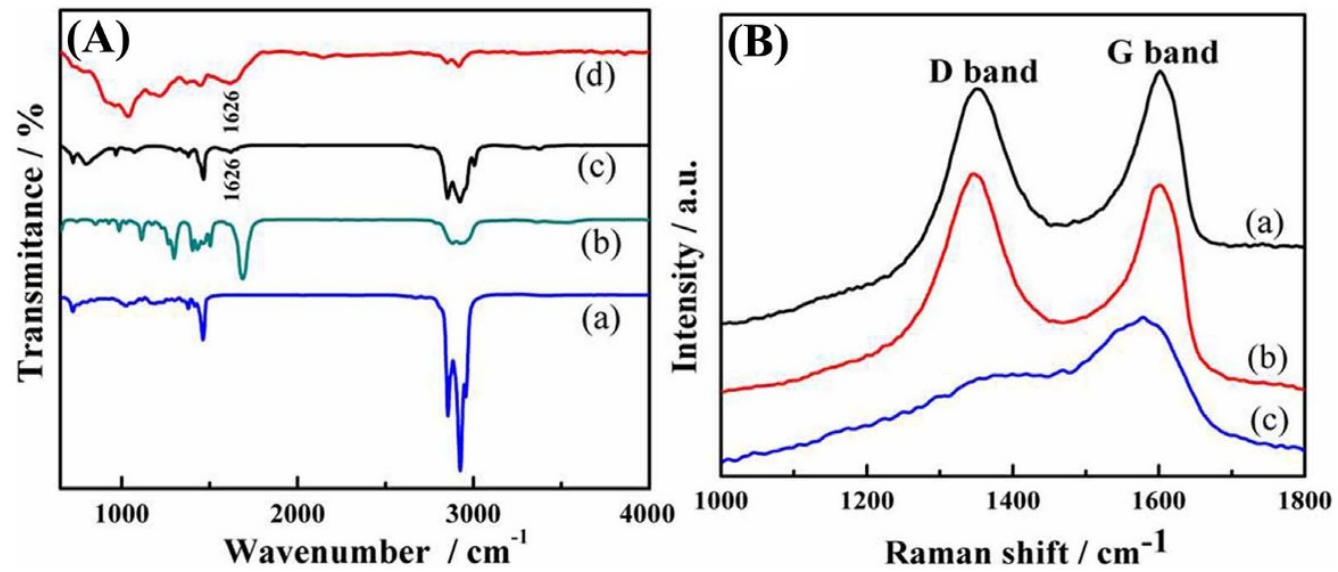

Figure 4 | (A) FT-IR spectra of TOP, NMP, OAm and, Pd. The results suggested that the OAm molecule was capped on Pd NPs only; (B) The Raman spectra of (a) GO (b) Pd/RGO (c) Pd. Raman spectra confirmed the formation of RGO sheets.

surface, resulting in the presence of the N XPS peak. This result is consistent with a previous publication ${ }^{20}$. This result could explain the presence of $\mathrm{N}$ in XPS and element analysis (Figure S6b) as well.

Figure 4A shows an FT-IR spectrum of the as prepared Pd NPs coated with the organic species, providing qualitative information about molecules capped on the surface of these NPs. For comparison, spectra of as-used OAm, TOP and NMP are also included, which are in good agreement with published data ${ }^{28-29}$. The spectrum recorded for Pd is similar to that of OAm but not TOP or NMP. For example, a band at $\sim 1626 \mathrm{~cm}^{-1}$ that is assigned to the $\mathrm{N}-\mathrm{H}$ bending vibration of amino groups appears in spectra of both the OAm and the Pd NPs ${ }^{30}$. These observations suggest that the organic residues finally capped on Pd NPs are only OAm, although both NMP and TOP were used in the synthesis. On the basis of the above discussion, it is believed that OAm may act as a capping agent on metal surface sites.

Raman spectroscopy is a powerful, nondestructive tool to characterize carbonaceous materials. Figure $4 \mathrm{~B}$ shows the Raman spectra of GO, Pd/RGO and Pd nanoparticles. The G band is related to the in-plane vibration of $\mathrm{sp}^{2}$-bonded carbon atoms, while the $\mathrm{D}$ band is associated with the vibrations of carbon atoms with the $\mathrm{sp}^{3}$ electronic configuration of disordered graphene. The $\mathrm{I}_{\mathrm{D}} / \mathrm{I}_{\mathrm{G}}$ ratio of our $\mathrm{Pd} /$ RGO, shown in Figure 4B-b, was found to be higher than that of the initial GO, revealing a substantial reduction of $\mathrm{sp}^{3}$-bonded carbon atoms and oxidized molecular defects ${ }^{31}$. In addition, such an enhancement has also been observed in examples of GO and carbon nanotubes decorated with metal NPs, indicating a probable chemical interaction or bond between the metal NPs and graphene due to carbon vacancies and defects that resulted from the chemical reduction of $\mathrm{GO}^{32-33}$. These results, combined with the X-ray powder diffraction (XRD) results, which are very consistent with the XPS data, further confirm the formation of RGO sheets. As for Pd NPs, the signals obtained are much weaker than those from our Pd/RGO composite, and the signals differ in other ways.

The electrocatalytic behaviors of Pd/RGO (with different amount of TOP) and $\mathrm{Pd} / \mathrm{XC}-72$ catalysts toward formic acid oxidation were characterized by cyclic voltammogram measurement in $0.25 \mathrm{M}$ $\mathrm{H}_{2} \mathrm{SO}_{4}+0.25 \mathrm{M} \mathrm{HCOOH}$ solution at a scan rate of $50 \mathrm{mV} / \mathrm{s}$. Figure 5A shows cyclic voltammogram curve of Pd/RGO (synthesized with different amount of TOP) and Pd/XC-72 catalysts. From figure $5 \mathrm{~A}-\mathrm{a}$, the oxidation peak for the formic acid oxidation located at $0.20 \mathrm{~V}$ in the positive scan directions is found in the Pd/RGO (with $0.4 \mathrm{~mL}$ TOP) catalyst electrode, and the corresponding peak current density is $32.1 \mathrm{~A} / \mathrm{m}^{2}$, which the electrocatalytic activity of $\mathrm{Pd} / \mathrm{RGO}$ (with $0.4 \mathrm{~mL}$ TOP) is higher than those for recently reported result and commercial $\mathrm{Pd} / \mathrm{C}$ catalysts ${ }^{6,34-35}$. From $5 \mathrm{~A}-\mathrm{b}$ and $5 \mathrm{~A}-\mathrm{c}$, it can be seen clearly that electrocatalytic activity of catalyst decreased when the amount of TOP molecule increased $(0.6 \mathrm{~mL})$ or decreased $(0.2 \mathrm{~mL})$. For $\mathrm{Pd} / \mathrm{XC}-7 \mathrm{NPs}$ catalyst (figure $5 \mathrm{~A}-\mathrm{b})$, the oxidation peak is at $0.24 \mathrm{~V}$ and the current density is $13.7 \mathrm{~A} / \mathrm{m}^{2}$. The current density of $\mathrm{Pd} / \mathrm{RGO}$ (with $0.4 \mathrm{~mL}$ TOP) for formic acid oxidation was nearly 2.3 times higher than that of $\mathrm{Pd} / \mathrm{XC}-72$ catalysts. On the other hand, the main peak for formic acid oxidation on the Pd/RGO (with
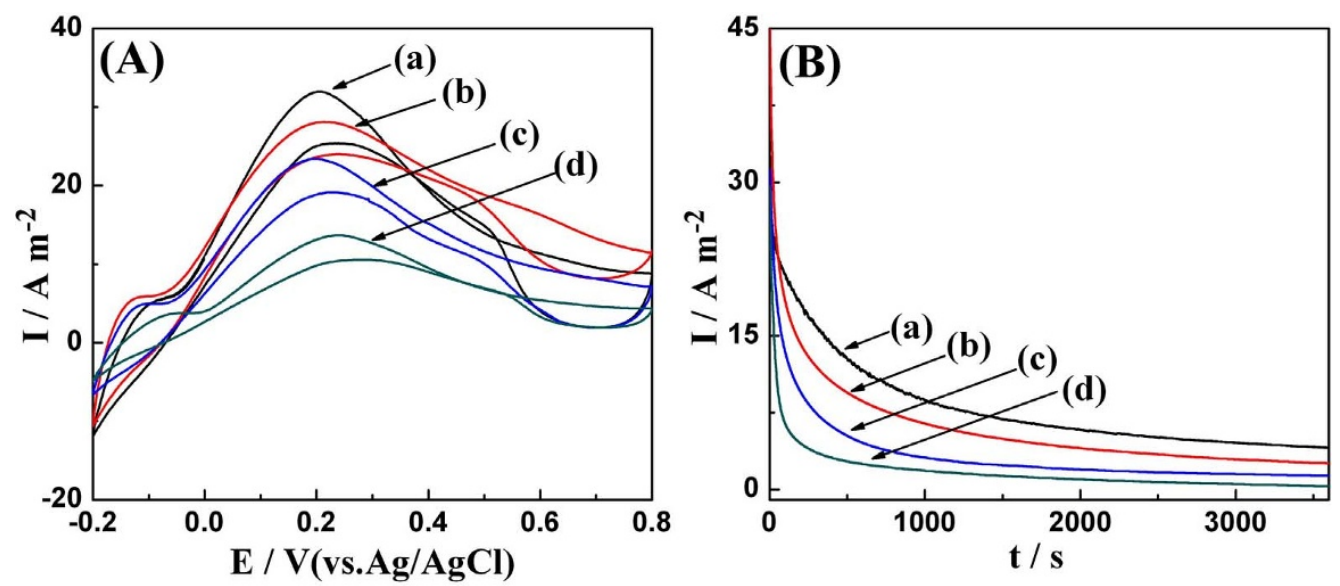

Figure $5 \mid$ (A) Cyclic voltammograms of different catalysts in $0.25 \mathrm{M} \mathrm{H}_{2} \mathrm{SO}_{4}+0.25 \mathrm{M} \mathrm{HCOOH}$ solution at a scan rate of $50 \mathrm{mV} / \mathrm{s}$; (B) Chronoamperometric curves of different catalysts in $0.25 \mathrm{M} \mathrm{H}_{2} \mathrm{SO}_{4}+0.25 \mathrm{M} \mathrm{HCOOH}$ solution at $0.1 \mathrm{~V}$ (vs. Ag/AgCl). The catalysts in the two panels are: (a) Pd/RGO(with $0.4 \mathrm{~mL}$ TOP), (b) Pd/RGO(with $0.2 \mathrm{~mL}$ TOP), (c) Pd/RGO(with $0.6 \mathrm{~mL}$ TOP), and (d) Pd/XC-72. 
$0.4 \mathrm{~mL}$ TOP) electrode shifted $40 \mathrm{mV}$ to lower potential direction, illustrating Pd/RGO NPs catalysts have higher electrocatalytic activity for formic acid oxidation than that of Pd/XC-72. The enhancement in catalytic activity of Pd/RGO NPs for formic acid oxidation can be attributed to more catalytic active sites from uniform shaped Pd NPs self-assembled on the RGO sheets and large surface area as well as good electrical conductivity of RGO sheets.

The higher formic acid oxidation activity of Pd/RGO was further confirmed by chronoamperometric measurements performed at $0.1 \mathrm{~V}$ (Figure 5B). Notably, Pd/RGO (with $0.4 \mathrm{~mL}$ TOP) showed both activity and durability superior to their Pd/RGO counterparts. It can be seen from the chronoamperometry curve, the limiting current densities of Pd/RGO (with $0.4 \mathrm{~mL}$ TOP) as catalyst for formic acid oxidation is higher than other catalysts. The electrooxidation current at $3600 \mathrm{~s}$ on $\mathrm{Pd} / \mathrm{RGO}$ (with $0.4 \mathrm{~mL}$ TOP) electrode is 13.7 times higher than that of the $\mathrm{Pd} / \mathrm{XC}-72$ electrode, which indicates that $\mathrm{Pd} / \mathrm{RGO}$ has a higher catalytic stability toward formic acid. This result, combined with the cyclic voltammetry (CV) measurements, further confirms the superior catalytic activity and stability of as-synthesized Pd/RGO for $\mathrm{HCOOH}$ oxidation.

\section{Discussion}

NMP is widely used in the chemical industry due to its cost-effectiveness and the ease with which it can be dissolved. The solvothermal reduction method for preparing RGO utilizes the high boiling point of NMP in combination with the oxygen-scavenging properties of NMP at high temperatures to deoxygenate $\mathrm{GO}^{36-37}$. In the reaction system, NMP was applied in an excessive amount to facilitate complete reduction. The essence of the NMP reduction method is the redox reaction between metal salts and NMP. In the redox process, the metal ions are reduced to the metallic state and nucleate to form NPs. At the same time the NMP probably are oxidized to organic compounds containing amide groups. To further confirm that, another experiment was carried out under the same conditions but with only $\mathrm{Pd}(\mathrm{acac})_{2}$ and NMP present. From the TEM images (Figure S7a), we can find that the Pd nanospheres have a mean diameter of $80 \mathrm{~nm}$. The selected-area electron diffraction (SAED) pattern (Figure S7b) of the Pd NPs exhibits several bright concentric rings, which can be attributed to $\{111\}$ (inner ring) and other crystal planes of the $f c c$ crystalline metal Pd. The XPS measurements also show the presence of $\mathrm{Pd}^{\circ}$ (Figure S6). Although the NMP method has been widely applied to synthesize RGO, the reduction capability of NMP has rarely been used to synthesize metallic NPs, and it is especially noteworthy here that the reaction mechanism has been studied little. The presence of an amide compound such as N-methylformamide has been detected ${ }^{38}$. The degree of NMP oxidation is mainly influenced by oxygen-free conditions of the reaction solutions.

In summary, we have reported here a comparatively easy, one-pot method to synthesize monodisperse palladium NPs, dispersed on RGO in NMP solution. Most importantly, the present study shows that $\mathrm{Pd} / \mathrm{RGO}$ has a much higher catalytic activity and better stability than conventional Pd/XC-72 electrocatalysts for formic acid electrooxidation. It is envisaged that this easy preparation method can readily be extended to the synthesis of other hybrids of metal nanoparticles and graphene for other heterogeneous catalyst applications.

\section{Methods}

Materials. Palladium acetylacetonate $\left(\mathrm{Pd}(\mathrm{acac})_{2}, 99 \%\right)$, sodium tetrachloropalladate (II) $\left(\mathrm{Na}_{2} \mathrm{PdCl}_{4}, 98 \%\right)$, palladium(II) acetate $\left(\mathrm{Pd}\left(\mathrm{C}_{2} \mathrm{H}_{3} \mathrm{O}_{2}\right)_{2}, 99 \%\right)$, trioctylphosphine (TOP, $90 \%$ ), oleylamine (OAm, $>70 \%$ ) and $N$-methyl-2-pyrrolidinone (NMP, ACS reagent grade) were all purchased from Sigma Aldrich. Graphite power (SP grade) was purchased from Sinopharm Chemical Reagent Co., Ltd., China. All chemical reagents were used without further purification.

Synthesis of the Pd/RGO composite. The graphite oxide was synthesized from natural graphite powder following the established practice ${ }^{39}$. Then, exfoliation of graphite oxide to graphene oxide (GO) was achieved in NMP solvent by ultrasonication of the dispersion for $1 \mathrm{~h}$. Finally, a homogeneous GO dispersion
(0.5 $\mathrm{mg} \mathrm{mL}^{-1}$ ) was obtained. Following a typical procedure for preparing $\mathrm{Pd} / \mathrm{RGO}$ under the protection of a nitrogen flow, $20 \mathrm{~mL}$ of GO-in-NMP solution $(0.5 \mathrm{mg}$ $\mathrm{mL}^{-1}$ ) was prepared in a flask. Next, $19.2 \mathrm{mg}$ of $\mathrm{Pd}(\mathrm{acac})_{2}$ was added into $0.2 \mathrm{~mL}$ of TOP which was then added to $5 \mathrm{~mL}$ of oleylamine and injected into the GO solution. The solution was heated to $60^{\circ} \mathrm{C}$ and kept at this temperature for $10 \mathrm{~min}$. Then it was heated up to $200^{\circ} \mathrm{C}$ for $2 \mathrm{~h}$. The resulting solution was cooled to room temperature, and then $30 \mathrm{~mL}$ ethanol was added to yield a black precipitate which was collected by centrifuging $(6000 \mathrm{rpm})$. The resulting black precipitate was dried completely at $50^{\circ} \mathrm{C}$. For comparison, the same procedure was followed to synthesize a $\mathrm{Pd} / \mathrm{XC}-72$ composite. In addition, $\mathrm{Pd}\left(\mathrm{C}_{2} \mathrm{H}_{3} \mathrm{O}_{2}\right)_{2}$ and $\mathrm{Na}_{2} \mathrm{PdCl}_{4}$ were used as two different palladium precursors to prepare two samples of $\mathrm{Pd} / \mathrm{RGO}$ composite under the same procedure.

Preparation of electrodes. Glassy carbon (GC) electrodes, $5 \mathrm{~mm}$ in diameter (electrode area $0.2 \mathrm{~cm}^{2}$ ), polished with $0.05 \mu \mathrm{m}$ alumina to a mirror-finish before each experiment, were used as substrates for supported catalysts. The catalysts were washed with isopropanol three times to remove excess surfactant. For the electrode preparation, typically, $3 \mathrm{mg}$ catalysts were added into $0.5 \mathrm{~mL} 0.05 \mathrm{wt}$. \% Nafion solution, and then the mixture was treated for $1 \mathrm{~h}$ with ultrasonication for uniform dispersion. A measured volume $(30 \mu \mathrm{L})$ of this mixture was dropped by a microsyringe onto the top surface of the GC electrode. The catalyst-modified GC electrode so obtained was employed as the working electrode in our experiments.

Characterization. Infrared spectra were recorded with a model 360 Nicolet AVATAR FT-IR spectrophotometer. X-ray photoelectron spectroscopy (XPS) was recorded on an ASTM E1829-02 system. Raman spectra (inVia, Renishaw, England) were used to study the integrity and electronic structure of the samples. X-ray diffraction (XRD) analysis was performed on a Bruker D8-ADVANCE diffractometer with $\mathrm{Cu}$ Ka radiation at a wavelength of $\lambda=0.15418 \mathrm{~nm}$. Transmission electron microscopy (TEM, JEOL JEM-2100) was used to investigate the morphology of samples. The crystalline properties of Pd/RGO were observed using high resolution TEM (JEOL JEM-2010).

Electrochemical measurements. All electrochemical measurements were carried out with a CHI $660 \mathrm{D}$ electrochemical workstation, using a three-electrode test cell. A conventional three-electrode system was used with a modified GC electrode as working electrode (5 $\mathrm{mm}$ in diameter), a Pt wire as counter electrode and a saturated calomel electrode (SCE) as reference electrode, respectively. Each working electrode contained about $0.18 \mathrm{mg} \cdot \mathrm{cm}^{-2}$ of the noble metal. All electrolytes were deaerated by bubbling $\mathrm{N}_{2}$ for $20 \mathrm{~min}$ and protected with a nitrogen atmosphere during the entire experimental procedure. All experiments were carried out at a temperature of $25 \pm$ $1{ }^{\circ} \mathrm{C}$.

1. Liu, H. Z. et al. Selective phenol hydrogenation to cyclohexanone over a dual supported Pd-lewis acid catalyst. Science 326, 1250-1252 (2009).

2. Nishihata, Y. et al. Self-regeneration of a Pd-perovskite catalyst for automotive emissions control. Nature 418, 164-167 (2002).

3. Michler, P. et al. Quantum correlation among photons from a single quantum dot at room temperature. Nature 406, 968-970 (2000).

4. Chen, Y. H. et al. Seed-Mediated synthesis of palladium nanorods and branched nanocrystals and their use as recyclable suzuki coupling reaction catalysts. J. Am. Chem. Soc. 131, 9114-9121 (2009).

5. Pacardo, D. B. et al. Biomimetic Synthesis of Pd Nanocatalysts for the Stille Coupling Reaction. ACS Nano 3, 1288-1296 (2009).

6. Mazumder, V. et al. Oleylamine-mediated synthesis of Pd nanoparticles for catalytic formic acid oxidation. J. Am. Chem. Soc. 131, 4588-4589 (2009).

7. Zhang, J. et al. Facile fabrication and unexpected electrocatalytic activity of palladium thin films with hierarchical architectures. J. Phys. Chem. C 112, 13970-13975 (2008).

8. Zhou, W. P. et al. Size effects in electronic and catalytic properties of unsupported palladium nanoparticles in electrooxidation of formic acid. J. Phys. Chem. B 110, 13393-13398 (2006).

9. Meng, H. et al. Electrosynthesis of Pd single-crystal nanothorns and their application in the oxidation of formic acid. Chem. Mater. 20, 6998-7002 (2008).

10. Guo, S. J. et al. Three-dimensional Pt-on-Pd bimetallic nanodendrites supported on graphene nanosheet: facile synthesis and used as an advanced nanoelectrocatalyst for methanol oxidation. ACS Nano 4, 547-555 (2010).

11. Geim, A. K. \& Novoselov, K. S. The rise of graphene. Nat. Mater. 6, 183-191 (2007).

12. Bunch, J. S. et al. Electromechanical resonators from graphene sheets. Science $\mathbf{3 1 5}$, 490-493 (2007).

13. Park, S. \& Ruoff, R. S. Chemical methods for the production of graphenes. Nat. Nanotechnol. 4, 217-224 (2009).

14. Sharma, S. et al. Rapid microwave synthesis of CO tolerant reduced graphene oxide-supported platinum electrocatalysts for oxidation of methanol. J. Phys. Chem. C 114, 19459-19466 (2010).

15. Hassan, H. M. A. et al. Microwave synthesis of grapheme sheets supporting metal nanocrystals in aqueous and organic media. J. Mater. Chem. 19, 3832-3837 (2009).

16. Pasricha, R. et al. A facile and novel synthesis of Ag-graphene-based nanocomposites. Small 5, 2253-2259 (2009). 
17. Xu, C., Wang, X. \& Zhu, J. W. Graphene-Metal Particle Nanocomposites. J. Phys. Chem. C 50, 19841-19845 (2008).

18. Paredes, J. I. et al. Graphene oxide dispersions in organic solvents. Langmuir 24, 10560-10564 (2008).

19. Pham, V. H. et al. Chemical functionalization of grapheme sheets by solvothermal reduction of agraphene oxide suspension in N-methyl-2-pyrrolidone. J. Mater. Chem. 21, 3371-3377 (2011).

20. Dubin, S. et al. A one-step, solvothermal reduction method for producing reduced graphene oxide dispersions in organic solvents. ACS Nano 4, 3845-3852 (2010).

21. Piao, Y. et al. Facile aqueous-phase synthesis of uniform palladium nanoparticles of various shapes and sizes. Small 3, 255-260 (2007).

22. Li, C. et al. Controllable polyol synthesis of uniform palladium icosahedra: effect of twinned structure on deformation of crystalline lattices. Angew. Chem. Int. Ed. 48, 6883-6887 (2009).

23. Sun, S. H. \& Murray, C. B. Synthesis of monodisperse cobalt nanocrystals and their assembly into magnetic superlattices. J. Appl. Phys. 85, 4325-4330 (1999).

24. Xu, Z. C. et al. Direct Colloidal Route for Pt-Covered AuPt Bimetallic Nanoparticles. J. Phys. Chem. Lett. 1, 2514-2518 (2010).

25. Shen, C. M. et al. Monodisperse noble-metal nanoparticles and their surface enhanced raman scattering properties. Chem. Mater. 20, 6939-6944 (2008).

26. Xu, D. et al. Solution-based evolution and enhanced methanol oxidation activity of monodisperse platinum-copper nanocubes. Angew. Chem. Int. Ed. 48, 4217-4221 (2009)

27. Saita, S. \& Maenosono, S. Formation mechanism of FePt nanoparticles synthesized via pyrolysis of iron(III) ethoxide and platinum(II) acetylacetonate. Chem. Mater. 17, 6624-6634 (2005).

28. Yang, H. T. et al. Stable cobalt nanoparticles passivated with oleic acid and triphenylphosphine. Nanotechnology 15, 70-74 (2004).

29. Xu, Z. et al. Oleylamine as both reducing agent and stabilizer in a facile synthesis of magnetite nanoparticles. Chem. Mater. 21, 1778-1780 (2009).

30. Zhang, J. \& Fang, J. Y. A general strategy for preparation of Pt 3d-transition metal (Co, Fe, Ni) nanocubes. J. Am. Chem. Soc. 51, 18543-18547 (2009).

31. Zhu, C. Z. et al. Reducing sugar: new functional molecules for the green Synthesis of graphene nanosheets. ACS Nano 4, 2429-2437 (2010).

32. Jasuja, K. \& Berry, V. Implantation and growth of dendritic gold nanostructures on graphene derivatives: electrical property tailoring and raman enhancement. ACS Nano 3, 2358-2366 (2009).

33. Lin, Y. et al. Rapid, solventless, bulk preparation of metal nanoparticle-decorated carbon nanotubes. ACS Nano 3, 871-884 (2009).

34. Hu, S. Z. et al. Electronic effect of Pd-transitionmetal bimetallic surfaces toward formic acid electrochemical oxidation. Electrochem. Commun. 38, 107-109 (2014)
35. Nassr, A. B. A. A. et al. Surfactant-free palladium nanodendrite assemblies with enhanced electrocatalytic performance for formic acid oxidation. Electrochimica Acta 102, 202-211 (2013).

36. Cai, M. F. \& Smart, R. B. Quantitative analysis of N-methyl-2-pyrrolidinone in coal extracts by TGA-FTIR. Energy Fuels 7, 52-56 (1993).

37. White, C. M. et al. Practical notes on the use of $N$-Methyl-2-pyrrolidinone as a solvent for extraction of coal and coal-related materials. Energy Fuels 11, 1105-1106 (1997).

38. Poulain, L. et al. Development of a new on-line mass spectrometer to study the reactivity of soluble organic compounds in the aqueous phase under tropospheric conditions: Application to $\mathrm{OH}$-oxidation of $\mathrm{N}$-methylpyrrolidone. J. Photochem. Photobiol. A: Chem. 187, 10-23 (2007).

39. Hummers, W. S. \& Offeman, R. E. Preparation of graphitic oxide. J. Am. Chem Soc. 80, 1339-1339 (1958).

\section{Acknowledgments}

The authors gratefully acknowledge the support of the National Basic Research Program of China (973 Program, Grant No. 2007CB209700 and 2013CBA01603).

\section{Author contributions}

Z.H.Y., C.M.S. and X.G.Z. designed experimentals. S.D.Y. and J.D. prepared samples and carried out characterization and electrochemical measurement. Y.T., X.Z.S. and S.X.L. contributed to the analysis and discussion for the results. S.D.Y., Z.H.Y., C.M.S. and X.G.Z. wrote the paper. All authors discussed the results and commented on the manuscript.

\section{Additional information}

Supplementary information accompanies this paper at http://www.nature.com/ scientificreports

Competing financial interests: The authors declare no competing financial interests.

How to cite this article: Yang, S.D. et al. One-Pot Synthesis of Graphene-Supported Monodisperse Pd Nanoparticles as Catalyst for Formic Acid Electro-oxidation. Sci. Rep. 4 4501; DOI:10.1038/srep04501 (2014).

\footnotetext{
cc) (i) $\Theta$ This work is licensed under a Creative Commons Attribution-

BY NC No NonCommercial-NoDerivs 3.0 Unported license. To view a copy of this license, visit http://creativecommons.org/licenses/by-nc-nd/3.0
} 